CORRECTION

https://doi.org/10.1038/s41586-018-0834-3

\title{
Publisher Correction: Enhanced strength and ductility in a high- entropy alloy via ordered oxygen complexes
}

Zhifeng Lei, Xiongjun Liu, Yuan Wu, Hui Wang, Suihe Jiang, Shudao Wang, Xidong Hui, Yidong Wu, Baptiste Gault,

Paraskevas Kontis, Dierk Raabe, Lin Gu, Qinghua Zhang, Houwen Chen, Hongtao Wang, Jiabin Liu, Ke An,

Qiaoshi Zeng, Tai-Gang Nieh \& Zhaoping Lu

Correction to: Nature https://doi.org/10.1038/s41586-018-0685-y, published online 14 November 2018.

In Fig. $1 \mathrm{~b}$ of this Letter, an error during the production process meant that all the data points (except the two points for O-2 and N-2, respectively) were missing. This figure has been corrected online. 